\title{
Design strategies for airline route maps, learning from the past
}

\author{
Menno-Jan Kraak ${ }^{\mathrm{a}, *}$, Paulo Raposo ${ }^{\mathrm{a}}$, \\ ${ }^{a}$ University of Twente /ITC,Menno-Jan Kraak-m.j.kraak@utwente.nl, Paulo Raposo - raposo@utwente.nl \\ * Corresponding author
}

Keywords: airline route maps, visual clutter, visualization strategies

\begin{abstract}
:
Airline route maps show the flights an airline operates between destination airports. Maps like these tend to become cluttered quickly. The clutter depends on the amount of flights to display; the routes of flight paths, the balance between shorter (e.g., domestic) and longer (e.g., intercontinental) flights, and the geographic locations of route endpoints each influence the amount of clutter.

Flights are typically shown as semantically uniform, despite having varied characteristics such as departure or arrival times, frequencies, or passenger volumes, among others. Most maps only indicate the existence of a connection since the main purpose is to impress the customers with the connectivity of the airline in question (i.e., the order or size of the network graph, corresponding to the number of locations served and the number of flight connections, respectively).

What are good strategies to design an airline route map? To answer this question, we looked at hundreds of airlines map from the past. From these maps we identified seven distinct strategies. Some rely on geometric transformations (i.e., typically warping or scaling, or the use of abstract space), while others use significant artistic freedom in symbol selection and design.
\end{abstract}

1. Projection Selection. A common strategy is to select a map projection that spreads or "unravels" the network. Figure A shows the JAL network in a polar azimuthal projection, in which regions farther away from the north pole are increasingly spread out, opening up map surface space for nodes, edges, and labels. Uncommon projection choices can help deal with networks containing routes that cross polar regions, or that have dense sets of connections in a region whose area the projection expands.

2. Topographic Manipulation. Segments of the map such as whole continents can be brought closer together, selectively scaled, or displaced to emptier areas of the map, to open up map surface space in a manner that is convenient for the network being drawn. In Figure B, Egypt has been conveniently moved into the Atlantic Ocean to allow for a more spread-out network of routes to locations in Europe. This strategy is frequently used when there are relatively spatiallydistinct but densely-packed clusters of endpoints in the network (e.g., multiple European and multiple North American destinations, but little elsewhere), or when moving one or a few topologically-central endpoints away from mass of its connecting nodes conveniently fans-out the network edges, such as in the provided Egyptian case.

3. Insets and Blow-Ups. The use of a main map for global connections and insets for smaller, busy areas or domestic networks is another frequently executed strategy. Figure C displays Pan Am's global network with insets for Europe and the eastern US. This strategy is particularly useful for networks that are "multiscale," in that their routes tend to cluster across short-haul, local tiers and long-haul, distant tiers.

4. Schematization. Schematic maps, where space is selectively distorted to produce relatively rectilinear or angular shapes, and exemplified by many public transport maps, are used as well. Usually, both the networks and the basemaps are schematized. Geographic distortions are varied, but generally ubiquitous. An example is found in Figure D, showing Air Canada's network. Schematization applies broadly, since geometric distortions can be selected at the designer's convenience, given the network in question.

5. Leaving Out Basemaps. Some airline route maps only show connections between destinations, leaving out any contextualizing basemap. The layout of network endpoints is not always consistent; they are sometimes planimetrically precise according to some map projection, and sometimes systematically or arbitrarily distributed across the map surface. The New York Air map in figure $\mathrm{E}$ is an example. Leaving out basemaps creates more whitespace on the map, which is either useful for drawing network information more densely, or left bare as an aesthetic choice. 
6. Abstract Diagrams. Finally, one can find non-map solutions, using diagrammatic techniques to visualize the network, even though these are frequently similar or evocative of maps. The characteristics of the geography in question, such as scale or directions, tend to be lost. Figure F show the connectivity of Turkish Airlines as a radial diagram; the globe in the center is decorative and suggestive only. For reasons similar to schematization, this strategy applies broadly, though it is interesting to note that it is relatively uncommon, perhaps because it tends to not produce maps.

7. Point Locations Only. A simple and minimalist approach sometimes taken is to plot destinations on a map while leaving out connection lines. As with leaving out basemaps, this creates more whitespace. Figure G shows an example for Air France.

The strategy applied appears to depend in part on the order and size of the network (i.e., number of endpoints and connections) and the geographic extent of the network. Smaller, more local airlines have less design challenges compared to global carriers.

How can we benefit from the existing design solutions described above to decide on a fitting design for illustrating any given airline's full network? Can we include and make use of further information from an airline's timetable in the map to visualize characteristics such as flight frequency, scheduling and duration?
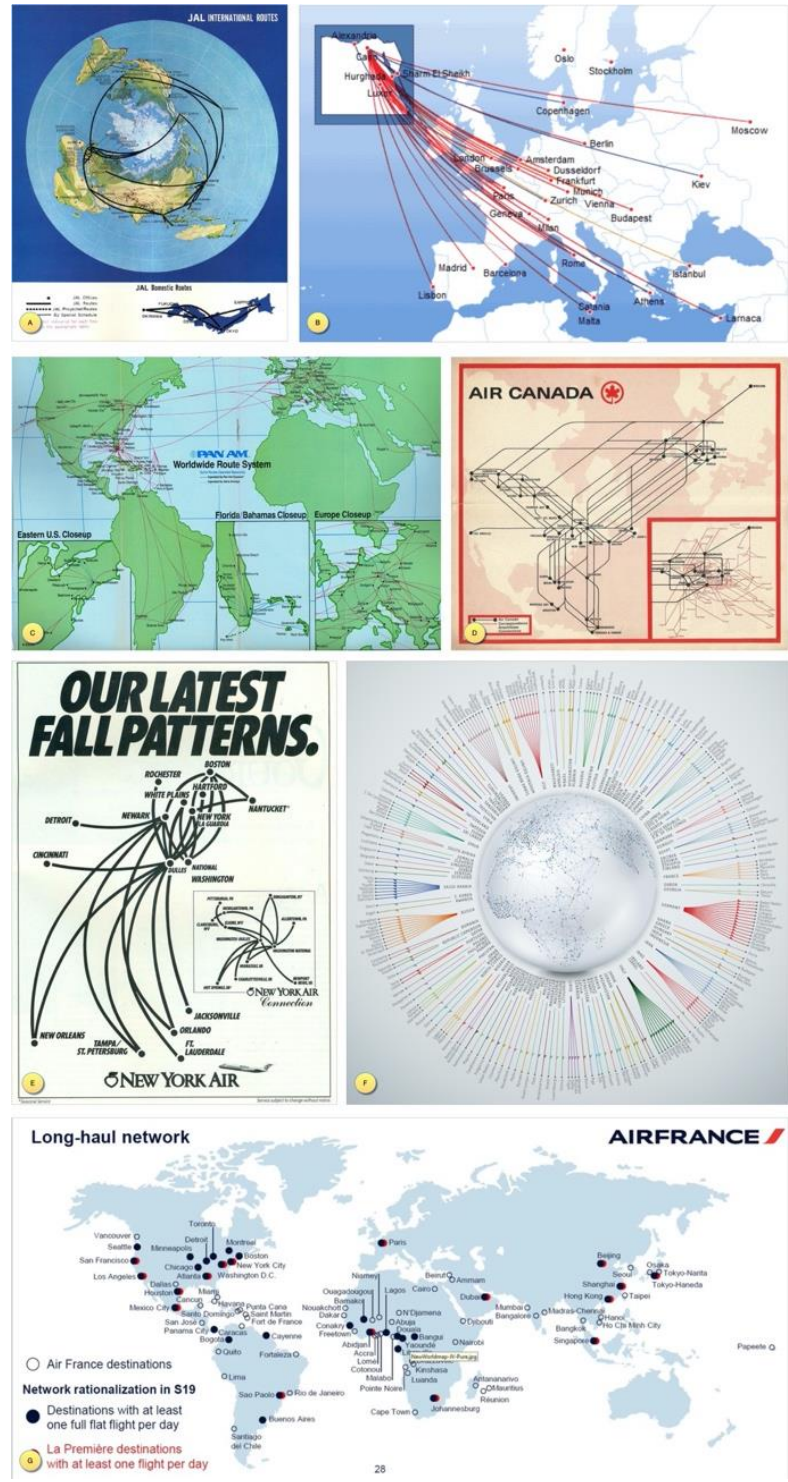

Figure 1. Airline network visualization strategies. 